\title{
Prevalence of Malocclusion and Orthodontic Treatment Modalities Among Mixed Indian Urban Population Seeking Treatment During 2016-2020 at Govt Zonal Hospital in Southern India: A Comprehensive Report
}

\author{
Akshai Kannan ${ }^{1}$, Vaibhav Jain ${ }^{1 *}$ and Merekhna Raghavan ${ }^{2}$ \\ ${ }^{1}$ Graded Specialist Orthodontics, INDC Danteshwari, Mumbai, Maharashtra, India \\ ${ }^{2}$ Senior Specialist Periodontist, Private Practice, Mumbai, Maharashtra, India \\ *Corresponding Author: Vaibhav Jain, Graded Specialist Prosthodontics, INDC \\ Danteshwari, Mumbai, Maharashtra, India.
}

Received: November 01, 2021

Published: November 30, 2021

(C) All rights are reserved by Akshai Kannan., et al.

\begin{abstract}
Aim: To study the pattern of distribution of different malocclusions and treatment modalities in the mixed Indian urban population at a Govt Zonal Hospital in Southern India.

Methods and Materials: This Descriptive cross-sectional study was conducted in 388 patients (188 were females and 200 were males) at a Dental Centre in a Zonal Govt Hospital in Southern India. The age criteria selected for the sample ranges between 6-46 years. The windows version 21 (SPSS Inc., Chicago, Illinois, USA) was used for statistical analysis and data prepared in MS excel sheet and was analysed. The Chi square test was used to compare the frequencies of categories of variables with groups.

Results: The data was collected from Jan 2016 to Dec 2020 on all OPD Days. Out of 388 patients, 188 were females and 200 were males. The mean age was 17.42 yrs. with SD of 6.525. There was a statistically significant/highly significant difference seen for the frequencies between the groups $(\mathrm{p}<0.01,0.05)$ with higher frequency for Class I Spacing being associated with Fixed mechanotherapy non extraction while Class I Crowding and Class II Div1, Class II Div2 and Class III with Fixed mechanotherapy with extraction.

Conclusions: The prevalence of malocclusion is high, a reason to continue training professionals to care for those patients in need of treatment. For further studies to asses prevalence and modalities of orthodontic treatment a well-distributed sample size for a nation with different ethnic groups distributed among India is recommended for planning orthodontic services at various Govt Zonal hospitals.
\end{abstract}

Keywords: Malocclusion; Orthodontic Treatment; OPD Registration

\section{Introduction}

The Study of distribution of malocclusion for its pattern and prevalence had been included in National Health surveys to get valuable information to plan and formulate the treatment facilities and the manpower in Orthodontic specialty in many countries
$[1,2]$. There are many studies to count on prevalence of malocclusion in different populations but only few of the studies based on review evaluated malocclusions in the referred population to make the plan and develop the treatment facilities depending on the pattern of the malocclusion and their treatment modalities [3]. The 
Zonal hospital has high need and demand for orthodontic treatment and no literature on modalities of orthodontic treatment and the distribution pattern of malocclusion. This research is formulated to study the pattern of distribution of different malocclusions and treatment modalities in the mixed Indian urban population at a Govt Zonal Hospital in Southern India.

\section{Material and methods}

This cross-sectional study was conducted in 388 patients (188 were females and 200 were males) at a Dental Centre in a Zonal Govt Hospital in Southern India. A written consent was signed by all patients before doing this survey and recording of the data. The age criteria selected for the sample ranges between 6-46 years. All the patients were of mixed Indian Urban population. The windows version 21 (SPSS Inc., Chicago, Illinois, USA) was used for statistical analysis and data prepared in MS excel sheet and was analysed. The $\mathrm{p}<0.05$ was found to be statistically significant for all the statistical tests, keeping $\alpha$ error at 5\% and $\beta$ error at 20\%, thus giving a power to the study as $80 \%$. The frequencies and percentage for categorical data, Mean and SD for numerical data, has been depicted. The Chi square test was used to compare the frequencies of categories of variables with groups.

\section{Results}

Out of 388 patients, 188 were females and 200 were males. The mean age was 17.42 yrs. with SD of 6.525 (Figure 1). Distribution of Malocclusion was Class I crowding (33.0\%) (Class II Div 1 (20.1\%), Class I Spacing (14.4\%), Class I Bimax (13.7\%), Class I Openbite (4.4\%), Class I Deepbite (3.1\%), Class Div 2 (3.4\%) and Class III (8.0\%) (Figure 2). The widely used treatment modality is Fixed mechanotherapy with extraction (78.4\%). The average treatment time 24.47 months with a SD of 8.176 (Figure 3). Comparison of Malocclusion and treatment indicates that the favourite treatment modality to treat Class I Crowding malocclusion was Fixed Mechanotherapy with extraction (122 patients) followed by Class II Div1 (62 patients) (Figure 4). The highly significant difference found for frequencies between the groups ( $p<0.01,0.05$ ) with higher frequency for Class I Spacing being associated with Fixed mechanotherapy non extraction while Class I Crowding and Class II Div1, Class II Div2 and Class III with Fixed mechanotherapy with extraction (Figure 5).

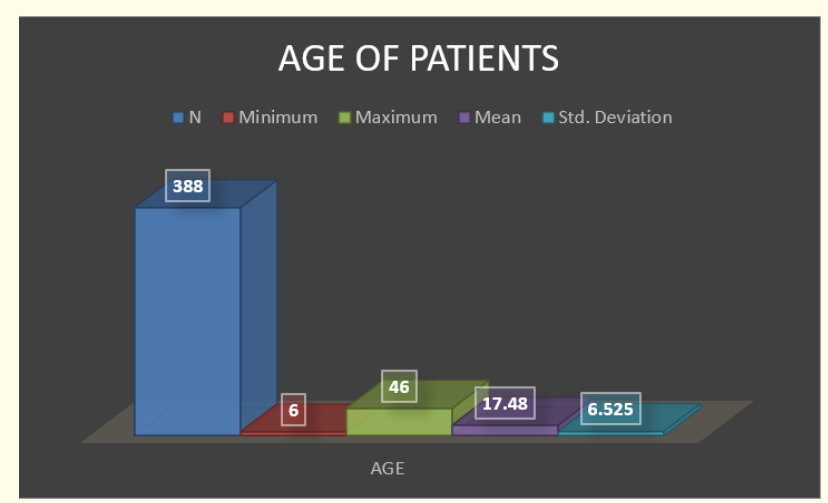

Figure 1

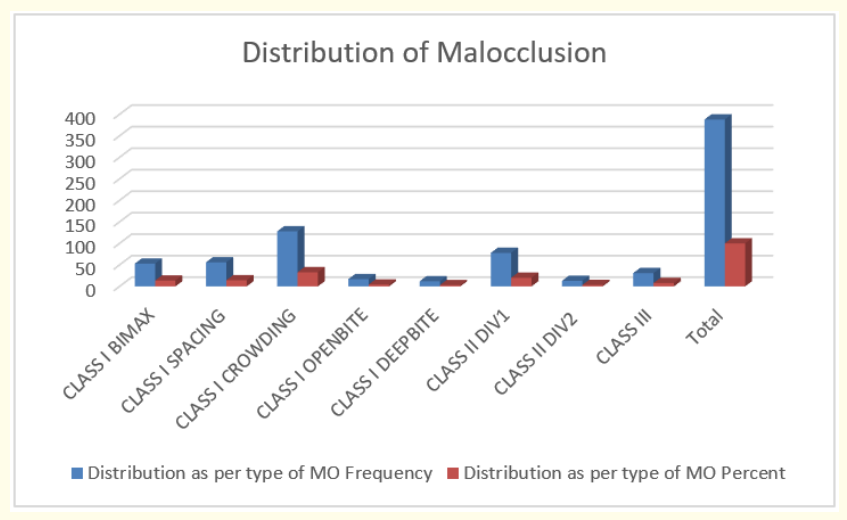

Figure 2

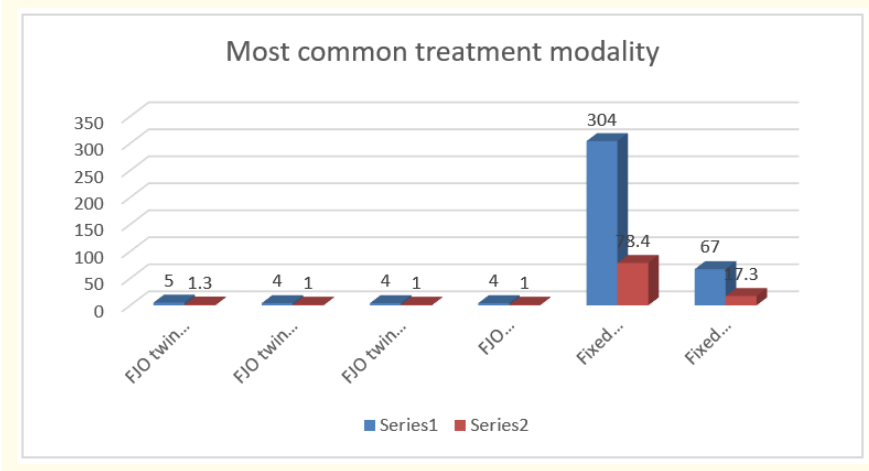

Figure 3 


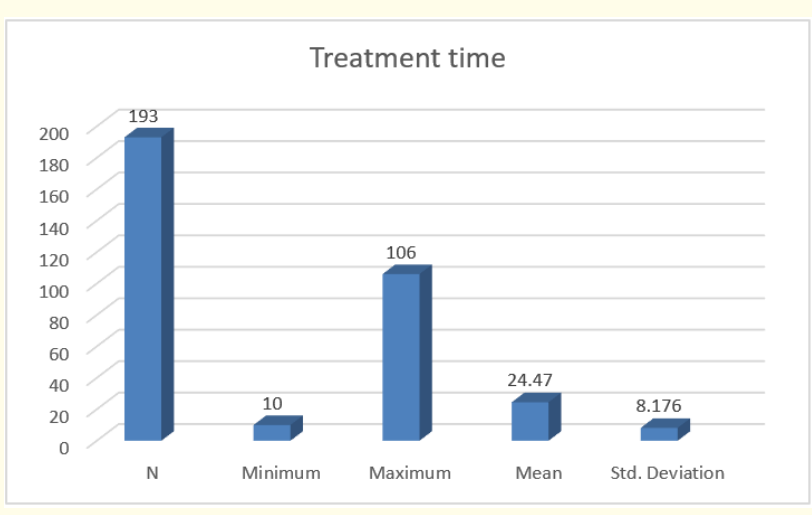

Figure 4

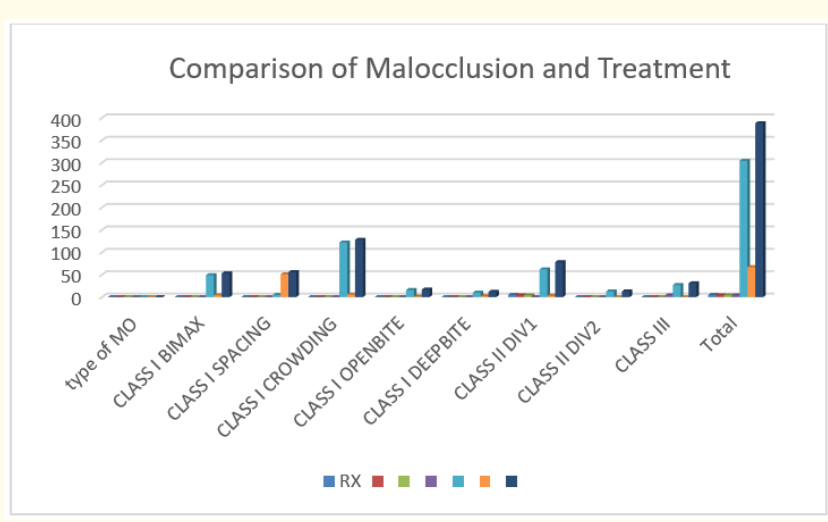

Figure 5

\section{Discussion}

The distribution of malocclusion usually varies depending on different race, origin and population. This type of study has been done to plan the orthodontic treatment which depend on need and pattern of distribution of malocclusion for patient benefit. Evaluation of malocclusion among the referred cases for orthodontic treatment and the various treatment modalities imparted has not been evaluated in a Mixed Indian population.

A similar study was carried out by Mohanty., et al. [4] in the Odisha population to assess the prevalence of malocclusion. From the study of 1207 subjects, 933 (77.3\%) subjects having Angle's Class I malocclusion and 252 (20.88\%) subjects were with Angle's Class
II malocclusion, whereas Angle's Class III malocclusion presented with only in 22 (1.82\%) subjects. Oomen., et al. [5] carried out a study in a similar setting with the prevalence of malocclusion to be 86.5\% with Angle's Class I malocclusion was found to be more distributed among the subjects as compared to the other types of malocclusions. Increased overjet in $81.5 \%$ of subjects was the major occlusal finding. This study shows the factors which help in making the decision to start orthodontic treatment and simultaneously decide data for planning orthodontic treatment service to service providers and clinicians with in the limited resources and allow to find out the implications of treatment.

A Study done by Min -Ho Jung [6] showed the malocclusion percentage distribution for male and female patients was $26.9 \%$ and $73.1 \%$ respectively. The same study also shown the age distribution percentage for the patients above having 13 years of age was $78.9 \%$, and above 19 years was $59.0 \%$. Approx 20.5 years is average age of whole patients. With regard to Angle classification, each percentage of Class I, Class II division 1, Class II division 2 and Class III malocclusion was $38.9 \%, 34.8 \%, 2.3 \%$ and $24.0 \%$. The percentage of extraction cases (60.9\%) outnumbered non extraction cases $(39.1 \%)$ and $46 \%$ of extraction cases were upper and lower 1st premolar extraction cases.

H Kaur [7] conducted a study for distribution of malocclusion that was done to record prevalence of malocclusion among 2,400 adolescents in state Karnataka, India and also differentiate malocclusion in rural and urban population. Some are school students with age group of 13-17 years, they are diagnosed for occlusal traits - sagittal occlusion, overjet, overbite, crowding, midline diastema, and crossbite. The study concluded that $87.79 \%$ of population had malocclusion. Out of which $89.35 \%$ had class I, $8.36 \%$ had class II, and $2.15 \%$ had class III malocclusion. Normal overjet and overbite were seen in 48.21 and $49.88 \%$ of subjects, respectively. The crowding was seen in $58.11 \%$ and $15.44 \%$ of subjects. Posterior crossbite in $0.99 \%$ and anterior crossbite was present in $8.48 \%$ and Urban population recorded twice the class II sagittal occlusion, and increased overjet as compared to rural population.

Akbari., et al. [8] conducted a meta-analysis on prevalence of malocclusion in Iranian school children. In total, 25 articles were included in the meta-analysis process. The dental malocclusion prevalence was estimated in 28,693 Iranian children aged 3-18 years. The total prevalence of Class I, II, and III malocclusion was 
54.6\% (46.5-62.7), 24.7\% (20.8-28.7), and 6.01\% (4-7.1), respectively. The prevalence of Class I, II, and III malocclusion was $44.6 \%$ (32.9-56.2), 21.5\% (18.01-25.1), and 4.5\% (3.2-5.9) in boys and $48.8 \%$ (36.8-60.8), 21.5\% (16.9-25.1), and 5.5\% (3.9-7.1) in girls, respectively. The meta-analysis was done aimed to estimate the prevalence of malocclusion in Iranian children. These results allow to shows the gravity of problem and given accurate plan for prevention and treatment of the problem.

Vibhute AH., et al. [9] conducted the study to determine the malocclusion incidence, characteristics and chief motivational factor among orthodontic patients for necessary treatment from State of Maharashtra, India. The dental casts were examined for pre-treatment records of 560 patients require orthodontic treatment includes 319 female and 241 male patients. The first upper and lower molars was examined for recording of occlusion according to the Angle's classification. Crowding, spacing, crossbite, overjet, overbite was recorded. The chief complaint of the patients was recorded from their history proformas. The study concluded Class I malocclusion was found in 274, Class II Division 1 in 196, Class II Division 2 in 57 and Class III malocclusion in 33 subjects. An increased overjet in 346 (61.7\%), crossbite was observed in 62 (11.07\%), and spacing was detected in 82 (14.6\%) patients. All patients reported that esthetics is the chief complaint for which they are seeking orthodontic treatment.

The similar study done by Rana., et al. [10] to assess the severity of malocclusion and orthodontic treatment require for 12-15-yearold school children of Patna, Eastern India. This was a cross-sectional study done by using Dental Aesthetic Index (DAI) index among 12-15-year-old school-going children to assess the severity of malocclusion. Among all, 902 children from middle schools (Government and Private) in Patna was selected. Around 5.3\% had a DAI score of $\leq 25$ which signifies that there is "no abnormality or may have minor malocclusion"; $15.3 \%$ had a DAI score of 26-30 which signifies that there is "definite malocclusion"; $6 \%$ had a DAI score of 31-35 which signifies that there is "severe malocclusion" and $4 \%$ had a DAI score of $\geq 36$ which signifies that there is "very severe or handicapping malocclusion". Among 14 years age group findings was more commonly seen. The study concluded that the prevalence of malocclusion around Patna state is reasonable high, it is also suggested that the awareness of malocclusion should be noticed at the school level as primary prevention can be the most effective tool in control this disease.
Our study tried to adopt the same guidelines in the studies mentioned above but with the inclusion of the factor of treatment modalities we were able to gauge the needs of the patient as well as encourage the patient to report earlier for orthodontic evaluation in order to attain the correct treatment modality.

\section{Conclusion}

From this hospital-based study, the following conclusions have been drawn: Most of the Mixed Indian urban Indian patients who requested clinical consultation regarding their malocclusion belong to an age group ranging from 6 to 46 years. Prevalence of malocclusion was found to be $33 \%$. Angle's Class I malocclusion with crowding was more prevalent as compared to the other types of malocclusions. 31\% of the studied population was treated by Fixed mechanotherapy with extraction. The indication of the majority of the patients reporting to the orthodontist at a later date rather than coming for a preliminary screening/mandatory screening from the age of 7 yrs. indicates that the awareness on orthodontic ailments (Malocclusion) is low with the Urban Indian population.

The vast majority of cases Angle's classification of malocclusion can also be utilized for clinically evaluating skeletal sagittal relationship. Identifying occlusal problems, their incidence and the need for treatment can help to determine the appropriate treatment plan and manpower needed in orthodontics.

The prevalence of malocclusion is high, a reason to continue training professionals to care for those patients in need of treatment. For planning orthodontic services for the people of India at various Govt Zonal hospitals a nationwide survey with well-distributed sample size considering different ethnic groups of India is recommended.

\section{Bibliography}

1. Migale D., et al. "Oral health and malocclusion in 10-11 yearsold children in southern Italy". European Journal of Paediatric Dentistry 10.1 (2009): 13-18.

2. Liu Z., et al. "The impact of malocclusion/orthodontic treatment need on the quality of life: a systematic review". The Angle Orthodontist 79.3 (2009): 585-591.

3. Prabhakar RR., et al. "Prevalence of malocclusion and need for early orthodontic treatment in children". Journal of Clinical and Diagnostic Research: JCDR 8.5 (2014): ZC60. 
4. Mohanty P., et al. "Pattern of malocclusion in orthodontic patients: A multi-centre study". Journal of International Oral Health 8.12 (2016): 1105.

5. Nainan 0., et al. "Evaluation of malocclusion pattern and dentofacial characteristics in orthodontically referred Urban Indians". Journal of Indian Orthodontic Society 47 (2013): 328-334.

6. Jung MH. "Current trends in orthodontic patients in private orthodontic clinics". Korean Journal of Orthodontics 39.1 (2009): 36-42.

7. Kaur H., et al. "Prevalence of malocclusion among adolescents in South Indian population". Journal of International Society of Preventive and Community Dentistry 3.2 (2013): 97.

8. Akbari M., et al. "Prevalence of malocclusion among Iranian children: A systematic review and meta-analysis". Dental Research Journal 13.5 (2016): 387.

9. Vibhute AH., et al. "Prevalence of malocclusion characteristics and chief motivational factor for treatment in orthodontic patients from Maharashtra, India". Journal of Orthodontic Research 1 (2013): 62-65.

10. Singh RN., et al. "Prevalence of malocclusion and orthodontic treatment needs among 12-15 years old school children in Patna, Eastern India". Journal of Family Medicine and Primary Care 8.9 (2019): 2983.

\section{Volume 5 Issue 12 December 2021}

(C) All rights are reserved by Akshai Kannan., et al. 